Revue internationale P.M.E.

Économie et gestion de la petite et moyenne entreprise

\title{
Vingt ans de recherche francophone en PME et entrepreneuriat
}

\section{Pierre-André Julien et Louis Raymond}

Volume 21, numéro 2, 2008

Vingt ans de recherche francophone en PME et entrepreneuriat

URI : https://id.erudit.org/iderudit/029429ar

DOI : https://doi.org/10.7202/029429ar

Aller au sommaire du numéro

Éditeur(s)

Presses de l'Université du Québec

ISSN

0776-5436 (imprimé)

1918-9699 (numérique)

Découvrir la revue

Citer cet article

Julien, P.-A. \& Raymond, L. (2008). Vingt ans de recherche francophone en PME et entrepreneuriat. Revue internationale P.M.E., 21(2), 7-8.

https://doi.org/10.7202/029429ar d'utilisation que vous pouvez consulter en ligne. 


\section{Éditorial}

\section{Vingt ans de recherche francophone en PME et entrepreneuriat}

Vingt ans pour les humains, c'est l'enfance qui disparaît, c'est la crise de l'adolescence estompée, c'est finalement le début de la maturité et d'une assurance croissante pour se tailler une place dans la vie.

Vingt ans pour la Revue internationale PME, c'est le souvenir de débuts difficiles, en 1988, pour s'assurer de publier des textes de qualité dans un nouveau domaine de recherche. L'idée était alors de faire auvre utile dans le milieu scientifique francophone pour susciter de plus en plus d'études sur les PME et l'entrepreneuriat, alors que ce domaine était encore considéré comme peu sérieux et que les lieux de recherche étaient rares. Il y avait bien quelques chercheurs isolés et quelques centres de formation en entrepreneuriat, notamment à Babson, aux États-Unis, mais rien de bien structuré. En 1988, on retrouvait quatre revues scientifiques sur le sujet, l'Internationales Gewerbearchiv - Zeitschrift für Klein- und Mittelunternehmen, créée en 1953 à Saint-Gall en Suisse, le Journal of Small Business Management, créée en 1963 à l'Université de Virginie occidentale, Entrepreneurship Theory and Practice, créée en 1976 à l'Université de Baylor au Texas sous le nom d'American Journal of Small Business et le Journal of Small Business \& Entrepreneurship, créée en 1983 à l'Université Ryerson, et dont la publication a été interrompue quelques années plus tard et reprise par la suite, mais rien de plus, montrant bien les limites de l'état de l'art en la matière.

En 1988, sans se concerter, des collègues voyaient en même temps la nécessité de multiplier ces lieux d'échanges et de stimulation de la recherche en $P M E$ et entrepreneuriat avec Entrepreneurship and Regional Development (créée d'abord à Dublin pour ensuite relever du Scandinavian Institute for Research and Entrepreneurship de l'Université de Växjö), Piccola Impresa de l'Université d'Urbino sous la gouverne d'Isa Marchini et l'année suivante le Journal of Business Venturing à Philadelphie. Nous faisions ainsi partie des pionniers puisque, depuis lors, plus de 15 autres revues ont vu le jour dans le monde, témoignant de l'intérêt croissant et finalement de la consécration de ce champ d'études malgré le refus de certains milieux conservateurs.

Derrière cette création, il y a d'abord eu le travail de Joseph Chicha, alors directeur du Département des sciences de gestion pour faire accepter en 1976 cet axe de recherche à l'Université du Québec à Trois-Rivières, puis les travaux de Michel Marchesnay et la création de l'Équipe de recherche sur la firme et l'industrie à l'Université de Montpellier I et, finalement, la collaboration de Robert Wtterwulghe de l'Université catholique de Louvain 
et ses contacts avec l'éditeur bruxellois De Boeck qui acceptait de produire les premiers numéros. Sur le premier conseil scientifique, on trouvait entre autres Ingolf Bamberger, Michel Capet, André Cibert, Laurent Creton, Yvon Gasse, Gérard Hirigoyen, Henri Le Marois, Henry Mahé de Boislandelle, Bernard Martory, Robert Paturel, Henri Pleitner, Jean Robidoux, Bertrand Saporta, Suzanne Savey, Jean-Marie Toulouse ou Sabine Urban, soit à peu près tous les chercheurs connus dans le milieu francophone qui travaillaient plus ou moins systématiquement dans ce domaine.

Nous lancions par la suite l'Association internationale de recherche en entrepreneuriat et PME (AIREPME) en 1996 lors du III Congrès international francophone en entrepreneuriat et PME (CIFEPME), tenu à Trois-Rivières à l'occasion du vingtième anniversaire du Groupe de recherche sur les PME. Cette association devait nous aider non seulement à consolider les échanges entre les congrès, mais également à stimuler l'envoi de manuscrits à la revue par de nouveaux auteurs ainsi que le recrutement de nouveaux évaluateurs.

Mais vingt ans, c'est aussi le début de la maturité avec un financement relativement assuré, des auteurs en quantité et plus de 400 abonnements, dont plus de 150 institutionnels pour les bibliothèques universitaires. Et cette maturité s'est réalisée en même temps que les travaux de l'OCDE sur l'entrepreneuriat et les PME auxquels nous avons été associés dès 1989 et conséquemment à la Charte de Bologne "sur les politiques à l'égard des PME» adoptée le 15 juin 2000 par 47 pays, à la déclaration ministérielle d'Istanbul du 3-5 juin 2004 sur «la promotion de la croissance de PME innovantes et compétitives sur le plan international » et, finalement, aux principes d'action adoptés à Brasilia pour le financement des PME et de l'entrepreneuriat les 27-30 mars 2006.

Toutefois, cette maturité, comme pour la fin de l'adolescence, n'est pas encore complétée. La concurrence est grande entre les revues et l'évolution de la science reste toujours imprévisible. Souhaitons-nous, à l'aube de la prochaine décennie de notre histoire, une plus grande assurance, mais aussi des défis pour continuer d'assumer notre rôle d'allumeur de réverbères, comme dans le Petit Prince, dans cette tâche toujours difficile et toujours à recommencer. Et c'est dans cet esprit qu'a été lancé, lors du IX CIFEPME à Louvain-la-Neuve en Belgique, un cédérom qui regroupe tous les articles publiés dans la Revue internationale PME de 1988 à 2007.

Enfin, nous osons espérer par ailleurs que le présent numéro de la revue poursuit cette quête d'excellence en recherche francophone sur les PME et l'entrepreneuriat.

Pierre-André Julien

Rédacteur en chef de 1988 à 2004

Louis Raymond

Rédacteur en chef

Revue internationale P.M.E., vol. 21, nº 2, 2008 